\title{
Real-world Management of Women with Postmenopausal Osteoporosis Treated with Denosumab: A Prospective Observational Study in the Czech Republic and Slovakia
}

\author{
Olga Růžičková (D) - Zdenko Killinger · Petr Kasalický • \\ Lisa Hamilton · Roman Tyl · Soňa Tomková · Lama Kalouche-Khalil
}

Received: April 6, 2018 / Published online: September 6, 2018

(C) The Author(s) 2018

\section{ABSTRACT}

Introduction: Osteoporosis is characterized by low bone mineral density (BMD) and an increased risk of fracture. In randomized controlled trials, denosumab has been shown to significantly reduce the fracture risk in women with osteoporosis. However, little is known about the real-world management of women who are prescribed denosumab.

Enhanced digital features To view enhanced digital features for this article go to https://doi.org/10.6084/ m9.figshare.6989195.

Electronic supplementary material The online version of this article (https://doi.org/10.1007/s12325018-0779-9) contains supplementary material, which is available to authorized users.

O. Růžičková (凶)

Institute of Rheumatology, Prague, Czech Republic

e-mail: ruzickova@revma.cz

O. Růžičková

Department of Rheumatology, 1st Faculty of

Medicine, Charles University, Prague, Czech

Republic

\section{Z. Killinger}

5th Department of Internal Medicine, Faculty of Medicine, Comenius University and University

Hospital, Bratislava, Slovakia

P. Kasalický

Bone Metabolism Unit, Affidea, Prague, Czech

Republic
Methods: This multicenter, prospective, observational real-world study in the Czech Republic and Slovakia evaluated the baseline characteristics and clinical management of women with postmenopausal osteoporosis prescribed denosumab for 24 months.

Results: A total of 600 women were included (300 in each country). In the Czech Republic and Slovakia, respectively, mean age at enrollment was 69.0 and 64.3 years, $67.7 \%$ and $30.0 \%$ of patients had a previous osteoporotic fracture, and $85.0 \%$ and $48.7 \%$ had previously received osteoporosis medication. In both countries, 'low BMD T score' and 'a history of osteoporotic fracture' were the main reasons for prescribing denosumab. Most patients received all four post-baseline denosumab injections (Czech Republic, 82.0\%; Slovakia, 81.0\%), and more

\section{Hamilton}

Amgen Ltd, Uxbridge, UK

R. Tyl

Private Rheumatology \& Osteology Department Osteomed, Trutnov, Czech Republic

\author{
S. Tomková \\ Department of Internal Medicine, P.J. Šafárik \\ University Košice, Hospital Košice-Šaca, Šaca, \\ Slovakia \\ L. Kalouche-Khalil \\ Amgen (Europe) GmbH, Zug, Switzerland
}


than $98 \%$ of patients in both countries received all injections at the prescribing center. At 24 months, most patients experienced an increase in BMD $T$ score for the lumbar spine, total hip, or femoral neck (Czech Republic, 69.7-91.7\%; Slovakia, 67.1-92.9\%). Adverse drug reactions were consistent with the known safety profile of denosumab.

Conclusion: Baseline characteristics of patients receiving denosumab in the Czech Republic and Slovakia reflect the reimbursement criteria for this agent in each country. The findings of our study in patients who are at high risk for fracture are consistent with the growing body of evidence demonstrating the effectiveness of denosumab in real-world clinical practice.

Trial Registration: ClinicalTrials.gov identifier, NCT01652690.

Funding: Amgen Inc.

Keywords: Clinical practice; Czech Republic; Denosumab; Osteoporosis; Postmenopausal; Slovakia

\section{INTRODUCTION}

Osteoporosis is a common disease, characterized by low bone mineral density (BMD), leading to fragile bones and an increased fracture risk [1]. An estimated 27.5 million people were living with osteoporosis in 27 European countries in 2010, including more than half a million people in the Czech Republic and almost a quarter of a million in Slovakia [1]. This is equivalent to $5.0 \%$ and $4.2 \%$ of the total population in each country, respectively [1].

Osteoporosis and the consequent fractures are associated with increased mortality and significant patient morbidity, including an increased risk of pain [2, 3] and disability [1, 4-7]. In 2010, an estimated 44,000 and 22,000 incident fractures occurred in women aged 50 years or older in the Czech Republic and Slovakia, respectively [7]. Osteoporosis also results in a substantial healthcare burden, driven primarily by the costs associated with fractures [7]. The economic burden of incident and previous osteoporotic fractures in 2010 was estimated at $€ 273$ million in the Czech Republic and $€ 107$ million in Slovakia, with incident fractures representing $60 \%$ and $71 \%$ of these costs, respectively [7]. Thus, there is a clear need to improve the management of osteoporosis in postmenopausal women in these countries to reduce the significant morbidity and social and economic costs associated with this disease.

Bisphosphonates are prescribed widely to treat osteoporosis [6, 8], but suboptimal persistence and adherence to treatment have been reported [9-12]. Denosumab, a fully human monoclonal antibody targeted against the receptor activator of nuclear factor kappa B ligand, is an alternative osteoporosis treatment indicated in postmenopausal women and in men who have an increased risk of fractures [13]. In a pivotal phase 3 study, denosumab (60 mg subcutaneously) administered every 6 months (Q6M) for 3 years in postmenopausal women resulted in a significant reduction in the risk of vertebral, non-vertebral, and hip fractures compared with placebo [14]. Additionally, in patients with suboptimal adherence to daily or weekly oral bisphosphonates, switching to denosumab was well tolerated and more effective at increasing BMD than switching to monthly oral bisphosphonate treatment [15-17]. Several studies have shown that persistence with and adherence to denosumab are high: $81-95 \%$ of patients persist with treatment at 1 year [18-21] and 91\% at 3 years [22]. Furthermore, persistence is 1.5-2.0 times higher with denosumab than with bisphosphonates $[12,19]$. Poor persistence and adherence to osteoporosis treatment have been shown to increase fracture risk [23]. Thus, the reduction in fracture risk reported in clinical trials of osteoporosis medications may not be achieved in real-world clinical practice without good adherence to and persistence with treatment.

Denosumab was approved for use in patients with postmenopausal osteoporosis in Europe in 2010 [13]. Following the approval and launch of a new pharmaceutical, assessment of its use in the real-world setting is recommended. The approved dose of denosumab is $60 \mathrm{mg}$ administered subcutaneously Q6M; it should only be administered by an individual who has been adequately trained. Patients prescribed denosumab should also receive concurrent calcium and vitamin D supplementation, unless contraindicated 
$[13,24,25]$. It should be noted that denosumab reimbursement criteria differ between the Czech Republic and Slovakia. In both countries, patients must have a baseline BMD $T$ score of -2.5 or lower at the femoral neck, lumbar spine, or total hip. For reimbursement in the Czech Republic, patients must also have experienced a previous osteoporosis fracture, be intolerant to other osteoporosis treatments, or have not responded to other available osteoporosis therapies [26]. In Slovakia, denosumab is reimbursed as first-line treatment in patients who have been diagnosed with osteoporosis by dual-energy X-ray absorptiometry (DXA) assessment of BMD or who have sustained an osteoporotic fracture. It is therefore not possible to directly compare the real-world management of patients with osteoporosis in the two countries.

\section{Study Aims}

This observational study was conducted to describe the patient characteristics and clinical management of women with postmenopausal osteoporosis who received denosumab $60 \mathrm{mg}$ Q6M as part of routine clinical practice in the Czech Republic and Slovakia during their first 2 years of treatment. This study assessed the initiation, administration, and duration of treatment with denosumab as well as the associated real-world treatment efficacy and safety profile.

\section{METHODS}

\section{Study Design}

This prospective observational study was conducted in 32 centers. In accordance with the ethical principles of the Declaration of Helsinki, the study protocol was approved by a central regulatory ethics committee in Slovakia and by local institutional ethics committees in the Czech Republic.

\section{Participants}

Women were considered eligible for inclusion in the study if they had a clinical diagnosis of postmenopausal osteoporosis, were prescribed denosumab $60 \mathrm{mg}$ Q6M for the treatment of osteoporosis, had received their first injection of denosumab $60 \mathrm{mg}$ within 8 weeks before study enrollment, and had provided written informed consent according to the local regulations of each country. Women were not eligible to take part if they were currently participating in, or had previously participated in, a clinical trial for denosumab, had taken part in any clinical or device trial within the previous 6 months, had any contraindication for denosumab treatment according to the locally approved product label, or had any disorder that, in the opinion of the investigator, compromised their ability to provide appropriate written informed consent.

The decision to treat a patient with denosumab $60 \mathrm{mg}$ was made independently of, and before, enrollment in the study. However, the prescription of denosumab, administration of the first injection, and provision of informed consent could occur during the same visit. The recruitment period was from 26 June 2012 to 15 May 2013, and the last patient's last visit to a treatment center was in May 2015. Data were collected on patients for up to 2 years from entry into the study, unless they withdrew from the study or were lost to follow-up. Baseline assessments were conducted up to 1 year before or within 91 days of the first denosumab injection.

A sample size of approximately 300 patients per country was planned, based on the probability of capturing patient-related characteristics with a prevalence of approximately $1 \%$ or more in the population. Investigators offered the opportunity to participate in the study to all patients treated with denosumab $60 \mathrm{mg}$ Q6M during the enrollment period until the desired number of patients was reached. This observational study did not alter the routine clinical management of patients.

\section{Study Sites and Data Collection}

The study was conducted at 32 centers (15 in the Czech Republic and 17 in Slovakia). After feasibility assessment, the study centers were chosen as representative of clinics providing care to women with postmenopausal 
osteoporosis in each country and region, regarding type (e.g., hospital, non-hospital) and location. Detailed information on the management of patients treated with denosumab and their clinical characteristics were collected at the initial visit and for up to 24 months from enrollment, either directly or from medical records; this included data on denosumab administration, the healthcare professionals who prescribed and administered denosumab, previous and current therapies, medical history (including history of fractures), comorbidities, adverse drug reactions related to denosumab (ADRs), and serious ADRs.

In the Czech Republic, annual DXA scanning is permitted under the reimbursement criteria. In the absence of an agreed gold-standard scanning interval, however, frequency can vary among centers (e.g., annually, biennially, or 1 year after treatment initiation and biennially thereafter). Therefore, BMD data, as measured by DXA before or during denosumab treatment, were only available according to routine practice in participating centers.

ADRs were coded using the Medical Dictionary for Regulatory Activities (version 14.1 or later). Data on physician-related factors, such as geographical region and specialty, were also collected.

\section{Statistical Analysis}

All analyses were conducted on the full analysis set, which consisted of all enrolled patients who met the inclusion criteria. Frequency distributions were described for categorical variables. Continuous variables were summarized as the number of non-missing values, mean, standard deviation, median, lower and upper quartiles, and minimum and maximum values. Missing data were left as missing, and no imputation was applied. To assess the clinical management patterns of patients with osteoporosis, we analyzed data for the proportions of women who received all four denosumab injections; received all denosumab injections at the initial prescribing center; were referred by the prescribing physician to another healthcare professional for continuation of care or follow-up care; had a radiologic bone assessment before the decision to treat with denosumab; had a radiologic bone assessment during treatment with denosumab; had osteoporosis-related laboratory investigations before the decision to treat with denosumab; and had osteoporosis-related laboratory examinations during treatment with denosumab. Data were also analyzed for the types of healthcare professionals who administered denosumab, where denosumab was administered (at or outside of the initial prescribing center), and the number of denosumab injections received by each patient during the 24-month follow-up period.

\section{RESULTS}

\section{Patient Characteristics}

Six hundred women who met the eligibility criteria were included in this study: 300 from 15 centers in the Czech Republic and 300 from 17 centers in Slovakia (Supplementary Fig. 1). All enrolled patients received at least one denosumab injection, as per routine clinical practice and independent of enrollment on the study. Patient baseline characteristics are presented in Table 1.

In the Czech Republic, the mean age of the women at enrollment was 69.0 years, the mean time since menopause was 20.7 years, the mean age at menopause onset was 48.3 years, and the mean time since diagnosis of postmenopausal osteoporosis was 5.3 years. Most patients had experienced a previous osteoporotic fracture $(67.7 \%)$, and approximately one-third (32.3\%) had experienced more than one fragility fracture at the time of enrollment. The majority of patients $(85.0 \%)$ had previously received treatment for osteoporosis, and approximately half (50.3\%) had previously received calcium and/or vitamin $\mathrm{D}$ supplements. The most common previous osteoporosis treatments were vitamin D supplements (49.3\%), calcium supplements $(48.0 \%)$, ibandronate $(40.3 \%)$, and alendronate $(38.0 \%)$. A total of $81.7 \%$ of patients had a BMD $T$ score of -2.5 or less at any measured site. 


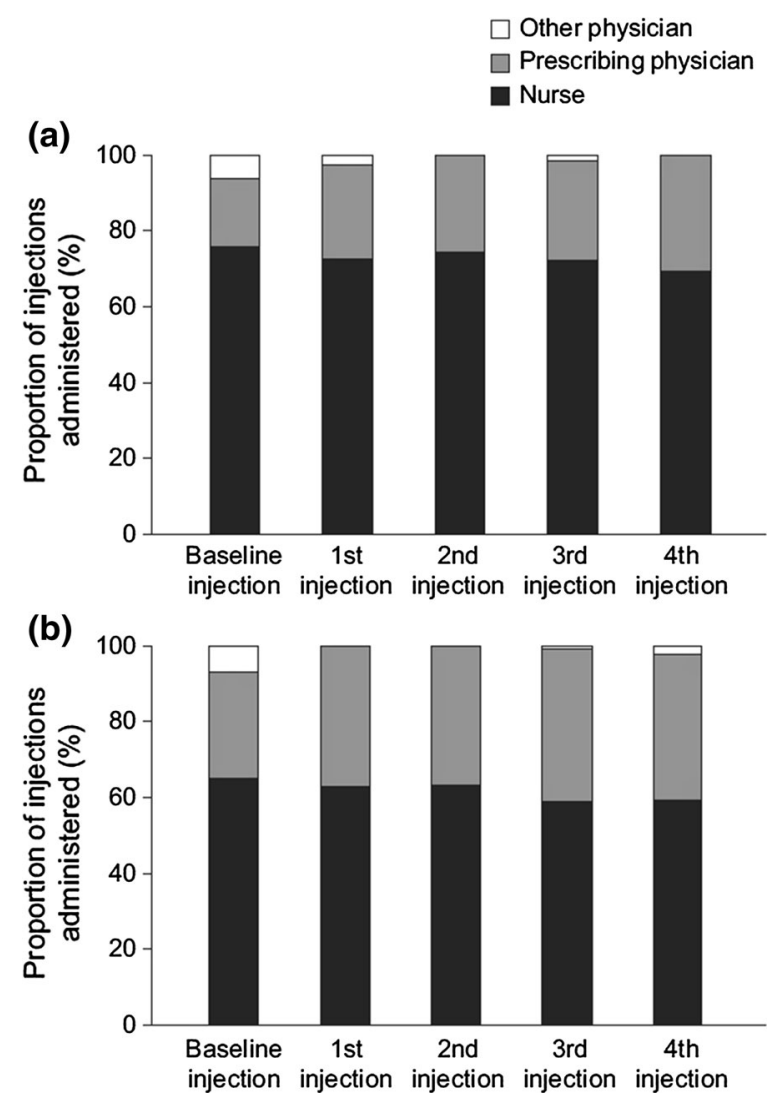

Fig. 1 Types of healthcare professional administering denosumab in a the Czech Republic and b Slovakia

In Slovakia, the mean age of participants at enrollment was 64.3 years, the mean time since menopause was 15.9 years, the mean age at menopause onset was 48.3 years, and the mean time since diagnosis of postmenopausal osteoporosis was 3.0 years. Approximately one-third $(30 \%)$ of patients had experienced an osteoporotic fracture prior to enrollment, but few $(7.0 \%)$ had experienced more than one. Approximately half $(48.7 \%)$ of patients had previously received an osteoporosis therapy; few $(6.3 \%)$ had previously received calcium and/or vitamin D supplements. The most common previous osteoporosis treatments were ibandronate $(18.0 \%)$, risedronate $(15.0 \%)$, strontium ranelate $(10.3 \%)$, and zoledronate $(10.0 \%)$. Overall, $76.7 \%$ of patients had a BMD $T$ score of -2.5 or less at any site.

\section{Prescribing Physicians and Study Centers}

In the Czech Republic, most prescribing physicians were either internists $(46.7 \%)$ or rheumatologists $(40.0 \%)$; the most frequent specialist type in Slovakia was rheumatologist (47.1\%). In both countries, the majority of prescribing physicians were male (Czech Republic, 73.3\%; Slovakia, 52.9\%). Most physicians had 10 or more years' experience (Czech Republic, 93.3\%; Slovakia, $88.2 \%)$. The majority of centers were not in hospitals (Czech Republic, 66.7\%; Slovakia, $58.8 \%$ ), and most were in urban areas (Czech Republic, 93.3\%; Slovakia, 100.0\%). In the Czech Republic, 93.3\% of centers were nonacademic and $6.7 \%$ were academic; in Slovakia, $58.8 \%$ of centers were non-academic, $23.5 \%$ were academic, and $11.8 \%$ were classified as both.

\section{Reasons for Prescribing Denosumab}

In the Czech Republic, the main reasons for prescribing denosumab were 'intolerant to other osteoporosis therapy' (53.3\%), 'low BMD $T$ score' $(46.0 \%)$, 'a history of osteoporotic fracture' (45.3\%), 'multiple risk factors for osteoporotic fracture' (42.0\%), and 'failed other available osteoporosis therapy' (36.3\%). In Slovakia, the main reasons for prescribing denosumab were 'low BMD $T$ score' (59.3\%), 'multiple risk factors for osteoporotic fracture' (20.0\%), 'a history of osteoporotic fracture' (18.3\%), 'failed other available osteoporosis therapy' (15.7\%), and 'intolerant to other osteoporosis therapy' (13.0\%). In total, $89 \%$ of patients treated in the Czech Republic met the reimbursement criteria for denosumab of: (1) having a baseline BMD $T$ score $\leq-2.5$ at the lumbar spine, total hip, or femoral neck and (2) having a history of osteoporotic fractures and/ or being intolerant to, or following failure of, other osteoporosis therapy. Data were not available to determine whether the remaining $11 \%$ of patients met the reimbursement criteria for denosumab in the Czech Republic. Equivalent data were not collected for individuals in Slovakia because these reimbursement requirements do not apply. 
Table 1 Patient baseline characteristics

\begin{tabular}{|c|c|c|c|}
\hline Characteristic & Czech Republic $(N=300)$ & Slovakia $(N=300)$ & Overall $(N=600)$ \\
\hline \multicolumn{4}{|l|}{ Age (years) } \\
\hline$n$ & 300 & 300 & 600 \\
\hline Mean (SD) & $69.0(8.7)$ & $64.3(8.6)$ & $66.7(9.0)$ \\
\hline \multicolumn{4}{|l|}{ Body mass index $\left(\mathrm{kg} / \mathrm{m}^{2}\right)$} \\
\hline$n$ & 296 & 268 & 564 \\
\hline Mean (SD) & $25.4(4.3)$ & $26.8(4.7)$ & $26.1(4.6)$ \\
\hline \multicolumn{4}{|l|}{ Age at menopause (years) } \\
\hline$n$ & 300 & 300 & 600 \\
\hline Mean (SD) & $48.3(5.6)$ & $48.3(5.2)$ & $48.3(5.4)$ \\
\hline Median (Q1, Q3) & $50.0(46.0,52.0)$ & $50.0(45.0,52.0)$ & $50.0(45.0,52.0)$ \\
\hline \multicolumn{4}{|l|}{ Years since menopause } \\
\hline$n$ & 300 & 300 & 600 \\
\hline Mean (SD) & $20.7(9.2)$ & $15.9(9.4)$ & $18.3(9.6)$ \\
\hline Median (Q1, Q3) & $21.0(13.5,28.0)$ & $15.0(9.0,22.0)$ & $17.0(11.0,25.0)$ \\
\hline \multicolumn{4}{|l|}{ Cause of menopause, $n(\%)$} \\
\hline Natural onset & $248(82.7)$ & $244(81.3)$ & $492(82.0)$ \\
\hline Clinically/surgically induced & $51(17.0)$ & $53(17.7)$ & $104(17.3)$ \\
\hline Unknown & $1(0.3)$ & $3(1.0)$ & $4(0.7)$ \\
\hline \multicolumn{4}{|l|}{ Time since PMO diagnosis (years) } \\
\hline$n$ & 300 & 300 & 600 \\
\hline Mean (SD) & $5.3(5.2)$ & $3.0(4.7)$ & $4.1(4.8)$ \\
\hline Median (Q1, Q3) & $4.7(0.7,8.2)$ & $1.0(0.1,4.6)$ & $2.6(0.2,6.8)$ \\
\hline Any historical fracture, $n(\%)$ & $221(73.7)$ & $100(33.3)$ & $321(53.5)$ \\
\hline Osteoporotic $^{\mathrm{a}}$ & $203(67.7)$ & $90(30.0)$ & $293(48.8)$ \\
\hline Vertebral & $93(31.0)$ & $21(7.0)$ & $114(19.0)$ \\
\hline Non-vertebral & $153(51.0)$ & $74(24.7)$ & $227(37.8)$ \\
\hline Major non-vertebral $^{\mathrm{b}}$ & $119(39.7)$ & $62(20.7)$ & $181(30.2)$ \\
\hline Hip & $18(6.0)$ & $5(1.7)$ & $23(3.8)$ \\
\hline At least two previous fractures, $n(\%)$ & $97(32.3)$ & $21(7.0)$ & $118(19.7)$ \\
\hline \multicolumn{4}{|c|}{ Hospitalized for osteoporotic fracture, $n(\%)$} \\
\hline Yes & $63(21.0)$ & $11(3.7)$ & $74(12.3)$ \\
\hline No & $237(79.0)$ & $289(96.3)$ & $526(87.7)$ \\
\hline
\end{tabular}


Table 1 continued

\begin{tabular}{llll}
\hline Characteristic & Czech Republic $(N=300)$ & Slovakia $(N=300)$ & Overall $(N=600)$
\end{tabular}

At least one fall in previous 12 months, $n$ (\%)

$\begin{array}{llll}\text { Yes } & 61(20.3) & 24(8.0) & 85(14.2) \\ \text { No } & 239(79.7) & 276(92.0) & 515(85.8)\end{array}$

At least one occurrence of immobility in the previous 12 months, $n$ (\%)

$\begin{array}{llll}\text { Yes } & 19(6.3) & 6(2.0) & 25(4.2) \\ \text { No } & 281(93.7) & 294(98.0) & 575(95.8)\end{array}$

Previous exposure to PMO therapy, $n$ (\%)

$\begin{array}{lccr}\text { Yes } & 255(85.0) & 146(48.7) & 401(66.8) \\ \text { No } & 45(15.0) & 154(51.3) & 199(33.2)\end{array}$

Exposure to PMO therapy in the previous 12 months, $n$ (\%)

$\begin{array}{llll}\text { Yes } & 240(80.0) & 119(39.7) & 359(59.8) \\ \text { No } & 60(20.0) & 181(60.3) & 241(40.2)\end{array}$

Previous exposure to calcium and/or vitamin D supplements, $n$ (\%)

$\begin{array}{llll}\text { Yes } & 151(50.3) & 19(6.3) & 170(28.3) \\ \text { No } & 149(49.7) & 281(93.7) & 430(71.7)\end{array}$

Secondary osteoporosis, $n(\%)$
Yes
$45(15.0)$
$23(7.7)$
$68(11.3)$
No
$255(85.0)$
277 (92.3)
$532(88.7)$
Presence of at least one comorbidity, $n$ (\%) 290 (96.7)
$272(90.7)$
$562(93.7)$
Modified Wolfe comorbidity index
$n$ 300
300
600
Mean (SD)
$1.9(1.5)$
$1.4(1.2)$
$1.6(1.4)$

$P M O$ postmenopausal osteoporosis, $Q$ quarter, $S D$ standard deviation

a Any fracture recorded on the clinical record form not including skull, facial bones, fingers, and toes and not associated with known high trauma severity or pathologic fractures

b A subset of non-vertebral fractures including those at the following locations: pelvis, hip, upper leg (not hip), lower leg (not knee or ankle), ribs, shoulder, forearm, and wrist and not associated with known high trauma severity or pathologic fractures

\section{Tests Performed Before and During Treatment with Denosumab}

Most patients (Czech Republic, 90.7\%; Slovakia, 91.7\%) had had at least one baseline DXA assessment, and $40.7 \%$ and $46.7 \%$, respectively, had had an assessment up to 3 months before the first denosumab injection. In addition, a DXA assessment had been performed at any time prior to prescription of denosumab in $99.0 \%$ of patients $(n=297)$ in the Czech Republic and $99.7 \% \quad(n=299)$ in Slovakia. 
Follow-up DXA assessments (after initiation of denosumab) were performed in $84.3 \%$ of patients $(n=253)$ in the Czech Republic and $72.0 \% \quad(n=216)$ in Slovakia. Osteoporosisrelated laboratory examinations were performed in a greater proportion of patients in Slovakia than in the Czech Republic both at baseline and during treatment. The percentage of patients with post-baseline osteoporosis-related laboratory examinations ranged from 51.9 to $66.4 \%$ in the Czech Republic and from 70.4 to $84.8 \%$ in Slovakia (Table 2).

\section{Treatment Discontinuation}

Few patients withdrew from the study: $11.7 \%$ of patients $(n=35)$ in the Czech Republic and $7.0 \%$ of patients $(n=21)$ in Slovakia (Supplementary Fig. 2). At the end of the study, no patients in the Czech Republic and two patients in Slovakia were referred to other healthcare professionals. In total, $14.3 \%$ of patients $(n=$ $43)$ in the Czech Republic and $11.3 \%(n=34)$ in Slovakia discontinued denosumab treatment.

\section{Denosumab Treatment}

Most patients received their baseline injection and all four post-baseline injections of denosumab during the 24-month observation period (Czech Republic, 82.0\%; $n=246$; Slovakia, $81.0 \% ; n=243$ ) (Table 3).

\section{Denosumab Administration}

More than $98 \%$ of patients in both countries received all their injections at the center that initially prescribed denosumab, irrespective of the total number of injections received on study. In both countries, denosumab injections were most commonly administered by a nurse or the physician who prescribed denosumab (Fig. 1). In the Czech Republic, considering all injections from baseline to the fourth injection post-baseline, $69.1-75.7 \%$ were administered by a nurse compared with $18.0-30.9 \%$ by the prescribing physician. In Slovakia, 58.7-65.0\% were administered by a nurse and $28.0-40.9 \%$ by the prescribing physician (Fig. 1).

\section{Bone Mineral Density}

Most patients [272 (90.7\%) in the Czech Republic; 275 (91.7\%) in Slovakia] had at least one baseline DXA assessment. In the Czech Republic, $48.3 \%$ of patients $(n=145)$ had a DXA measurement at both baseline and 24 months at the lumbar spine, total hip, and femoral neck. In Slovakia, $51.7 \%$ of patients $(n=155)$ had a DXA measurement at baseline and 24 months at the lumbar spine and femoral neck, and $46.3 \%(n=139)$ had measurements at both time points at the total hip.

In the Czech Republic, mean BMD $T$ scores at baseline were $-2.74,-1.98$, and -2.16 at the

Table 2 Number and timing of osteoporosis-related laboratory examinations performed before and during treatment

\begin{tabular}{|c|c|c|c|c|c|c|}
\hline \multirow[t]{2}{*}{ Timing of examination } & \multicolumn{2}{|c|}{ Czech Republic } & \multicolumn{2}{|l|}{ Slovakia } & \multicolumn{2}{|l|}{ Overall } \\
\hline & $\overline{n / N}$ & $\%(95 \% \mathrm{CI})$ & $n / N$ & $\%(95 \% \mathrm{CI})$ & $n / N$ & $\%(95 \% \mathrm{CI})$ \\
\hline Baseline injection & $230 / 300$ & $76.7(71.5-81.3)$ & $260 / 300$ & $86.7(82.3-90.3)$ & $490 / 600$ & $81.7(78.3-84.7)$ \\
\hline First injection & $148 / 285$ & $51.9(46.0-57.9)$ & $207 / 294$ & $70.4(64.8-75.6)$ & $355 / 579$ & $61.3(57.2-65.3)$ \\
\hline Second injection & $162 / 280$ & $57.9(51.8-63.7)$ & $224 / 286$ & $78.3(73.1-83.0)$ & $386 / 566$ & $68.2(64.2-72.0)$ \\
\hline Third injection & $163 / 270$ & $60.4(54.3-66.2)$ & $226 / 280$ & $80.7(75.6-85.2)$ & $389 / 550$ & $70.7(66.7-74.5)$ \\
\hline Fourth injection & $174 / 262$ & $66.4(60.3-72.1)$ & $223 / 263$ & $84.8(79.9-88.9)$ & $397 / 525$ & $75.6(71.7-79.2)$ \\
\hline
\end{tabular}

CI confidence interval 

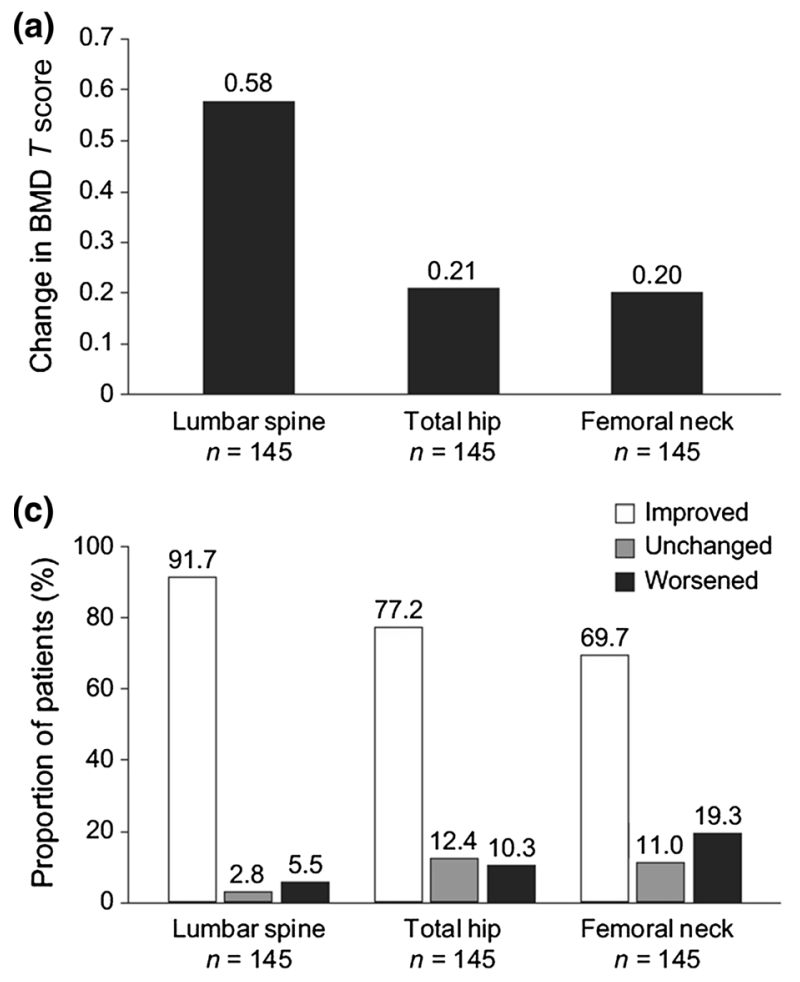

Fig. 2 Mean change in bone mineral density (BMD) $T$ score from baseline at 24 months in a the Czech Republic and $\mathbf{b}$ Slovakia and the proportion of patients with improved, unchanged, or worsened osteoporosis at 24 months in $\mathbf{c}$ the Czech Republic and d Slovakia;

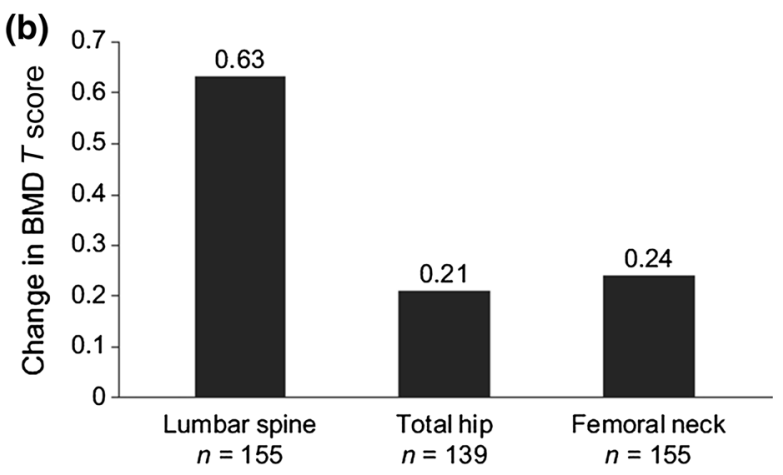

(d)
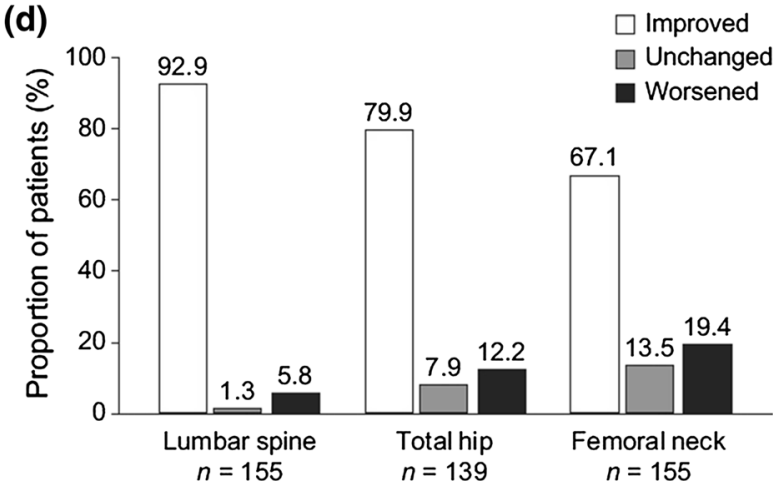

improvement was defined as an increase in BMD $T$ score $>0$, worsened was defined as a decrease in BMD $T$ score $<0$, and unchanged was defined as no change in BMD $T$ score

Table 3 Number of denosumab injections received following the baseline injection

\begin{tabular}{lccc}
\hline $\begin{array}{l}\text { Number of injections } \\
\text { post-baseline }\end{array}$ & $\begin{array}{l}\text { Czech Republic } \\
(\boldsymbol{N}=\mathbf{3 0 0})\end{array}$ & Slovakia $(\boldsymbol{N}=\mathbf{3 0 0})$ & Overall $(\boldsymbol{N}=\mathbf{6 0 0})$ \\
\hline 0 & $17(5.7)$ & $7(2.3)$ & $24(4.0)$ \\
1 & $7(2.3)$ & $10(3.3)$ & $17(2.8)$ \\
2 & $14(4.7)$ & $7(2.3)$ & $21(3.5)$ \\
3 & $16(5.3)$ & $33(11.0)$ & $49(8.2)$ \\
4 & $246(82.0)$ & $243(81.0)$ & $489(81.5)$ \\
\hline
\end{tabular}

Values are $n(\%)$

lumbar spine, total hip, and femoral neck, respectively, and $-2.19,-1.77$, and -1.96 at 24 months, respectively (Table 4). In Slovakia, mean BMD $T$ scores at baseline were -2.57 , -1.28 , and -1.87 at the lumbar spine, total hip, and femoral neck, respectively, and - 1.91, -1.07 , and -1.67 at 24 months, respectively (Table 4).
The mean change in BMD $T$ score from baseline to month 24 is shown in Fig. 2 . In both countries, the change was greatest at the lumbar spine. The mean change in BMD $T$ score at the total hip was the same for the two countries, and changes at the lumbar spine and femoral neck were similar between countries. At 24 months, the majority of patients in both 
Table 4 Mean (standard deviation) bone mineral density $T$ scores at baseline and 24 months

\begin{tabular}{llll}
\hline & Czech Republic $(\boldsymbol{N}=\mathbf{3 0 0})$ & Slovakia $(\boldsymbol{N}=\mathbf{3 0 0})$ & Overall $(\boldsymbol{N}=\mathbf{6 0 0})$ \\
\hline Lumbar spine & $n=268$ & $n=273$ & $n=541$ \\
Baseline & $-2.74(1.03)$ & $-2.57(0.85)$ & $-2.66(0.95)$ \\
& $n=147$ & $n=160$ & $n=307$ \\
Month 24 & $-2.19(1.0)$ & $-1.91(0.97)$ & $-2.04(0.99)$ \\
Total hip & $n=263$ & $n=246$ & $n=509$ \\
Baseline & $-1.98(0.84)$ & $-1.28(0.92)$ & $-1.64(0.94)$ \\
& $n=146$ & $n=151$ & $n=297$ \\
Month 24 & $-1.77(0.9)$ & $-1.07(0.85)$ & $-1.41(0.94)$ \\
Femoral neck & $n=262$ & $n=273$ & $n=535$ \\
Baseline & $-2.16(0.77)$ & $-1.87(0.82)$ & $-2.02(0.81)$ \\
& $n=146$ & $n=160$ & $n=306$ \\
Month 24 & $-1.96(0.77)$ & $-1.67(0.8)$ & $-1.81(0.8)$ \\
\hline
\end{tabular}

countries (for whom baseline and 24-month DXA assessments were available) experienced improvements in osteoporosis (an increase in BMD $T$ score $>0$ ): lumbar spine, $91.7 \%$ and 92.9\% of patients in the Czech Republic and Slovakia, respectively; total hip, $77.2 \%$ and 79.9\%; femoral neck, $69.7 \%$ and $67.1 \%$, respectively (Fig. 2).

\section{Adverse Drug Reactions}

In total, $2.7 \%$ of patients $(n=16)$ reported an ADR: $1.3 \%$ in the Czech Republic $(n=4)$ and $4.0 \%$ in Slovakia $(n=12)$. Of these, only one individual $(0.2 \%)$, in Slovakia, reported a serious ADR (myocardial infarction, which was resolved within 1 week). ADRs in patients in the Czech Republic were musculoskeletal pain $(0.7 \% ; n=2)$, headache $(0.3 \% ; n=1)$, and skin infection $(0.3 \% ; n=1)$. ADRs in patients in Slovakia were alopecia $(0.7 \% ; n=2)$, rash $(0.7 \%$; $n=2)$, hypocalcemia $(0.7 \% ; n=2)$, back pain
$(0.3 \% ; n=1)$, myocardial infarction $(0.3 \% ; n=$ $1)$, supraventricular tachycardia $(0.3 \% ; n=1)$, upper abdominal pain $(0.3 \% ; n=1)$, gingival swelling $(0.3 \% ; n=1)$, burning sensation $(0.3 \%$; $n=1)$, pyrexia $(0.3 \% ; n=1)$, dysuria $(0.3 \% ; n=$ $1)$, and dyspnea $(0.3 \% ; n=1)$. None of the ADRs were fatal. Seven patients $(1.2 \%)$ discontinued treatment because of ADRs.

New fractures were reported in $6.0 \%$ of patients $(n=16)$ in the Czech Republic and $1.3 \%(n=4)$ in Slovakia. In the Czech Republic, one atypical femoral fracture was reported and positively adjudicated. The fracture occurred approximately 2 years after receiving the first dose of denosumab. This patient was 79 years of age and had slipped on the floor and fallen from a standing position. The fracture was confirmed by radiography, which showed a femoral midshaft fracture. The patient had received bisphosphonates before starting denosumab therapy. Concomitant medications included calcium and vitamin D supplements and solifenacin succinate. Radiography performed 
within 6 months of the fracture confirmed that it had healed, and the patient was able to walk without assistance. The authors, including the treating physician, did not consider that the fracture was related to denosumab treatment.

\section{DISCUSSION}

In this 24-month real-world observational study of postmenopausal women with osteoporosis, conducted in the Czech Republic and Slovakia, most patients were prescribed denosumab according to the reimbursement criteria in their country. In both countries, denosumab was always administered by a healthcare professional, treatment discontinuation was low, as was the incidence of new fractures, and no new safety issues were identified.

The baseline characteristics and patient outcomes (specifically BMD) in this real-world study reflect those of the broad population of women with osteoporosis, some of whom are often excluded from clinical trials. There were some notable differences in baseline characteristics between patients from the two countries; patients in the Czech Republic were older, had a higher prevalence of previous osteoporotic fracture, were more likely to have previously received an osteoporosis medication, and had lower mean BMD $T$ scores. This most likely reflects differences in the reimbursement criteria for denosumab between the two countries. Indeed, for most patients, the reasons for prescribing denosumab were in line with the local reimbursement criteria, namely 'low BMD $T$ score,' 'a history of osteoporotic fracture,' 'multiple risk factors for fracture,' 'failure to respond to other available osteoporosis therapy,' and 'intolerance to other osteoporosis therapy.' The fact that DXA assessments were performed in at least $99 \%$ of patients prior to prescription of denosumab suggests that the results of these tests influence the treatment decision. Indeed, in the Czech Republic, DXA criteria for diagnosis and treatment must be fulfilled before treatment for osteoporosis is started.

A further $72-84 \%$ of women also had DXA scans while receiving denosumab, indicating that, in both countries, many patients continued to be monitored for changes in BMD during treatment. In total, $89 \%$ of patients in the Czech Republic met the reimbursement criteria for denosumab. Equivalent data were not collected in Slovakia because these reimbursement requirements do not apply.

Treatment discontinuation rates (11.3-14.3\%) were slightly higher than study withdrawal rates $(7.0-11.7 \%)$ but were relatively low overall; most individuals in both countries received all four post-baseline injections of denosumab $(82.0 \%$ and $81.0 \%$ of patients in the Czech Republic and Slovakia, respectively). The relatively low discontinuation rates observed in the real-world clinical settings are important because, while many patients with osteoporosis are treated with bisphosphonates, results from systematic reviews show that persistence with and adherence to therapy with these agents can be suboptimal $[9,10]$. Adherence to bisphosphonates and osteoporosis treatments has also been shown to be poor in studies carried out in postmenopausal women in the Czech Republic [27] and Slovakia [28]. Thus, denosumab may help to improve persistence with therapy compared with bisphosphonates in the real-world setting in these countries. Nevertheless, persistence-enhancing strategies should still be used to optimize medication-taking and clinical outcomes. Results of further studies examining such strategies would be of great interest.

Irrespective of the total number of injections received on study, more than $98 \%$ of patients in both countries received all their denosumab injections at the center where the initial injection was prescribed, suggesting similarities in management practices in the two countries. Regular contact with healthcare professionals at the prescribing center may have contributed to the low discontinuation rates in this study. It has previously been shown that regular interaction with nurses improves persistence with osteoporosis therapy $[29,30]$.

Persistence with denosumab has been reported to be high: in retrospective and prospective studies, $81-95 \%$ of patients were persistent with denosumab after 1 year of treatment and $75-86 \%$ were persistent after 2 years [18-21, 31]. However, an analysis of 
prescription data from the General Health Insurance Company of the Czech Republic showed that, although $93.8 \%$ of postmenopausal women with osteoporosis were compliant with denosumab treatment (defined as a medication possession ratio $\geq 0.8$ ), persistence (defined as a refill gap $\leq 30$ days) was only $59.1 \%$ after 12 months and $34.8 \%$ after 24 months [32]. The disparity between the persistence rate reported from the retrospective database study and the high proportion of women receiving all four doses of denosumab in our study may, in part, be explained by differences in study design. The 30-day refill gap used to define persistence in the retrospective database study could be considered restrictive. Previous retrospective and prospective studies of persistence with denosumab have used a 60-day or 8-week refill gap [18-21]; therefore, a refill gap longer than 30 days may be appropriate for assessing denosumab persistence. Our study measured discontinuation rather than persistence so refill gaps are not relevant. In addition, the prospective design of our study may have led to more rigorous recording of medicationtaking behavior; furthermore, patients were aware that they were being observed, which may have influenced their behavior [33].

The rate of new fractures in Slovakia in our study $(1.3 \%)$ was in line with the rate reported during clinical trials of denosumab in postmenopausal women with osteoporosis in which the proportion of patients experiencing new fractures ranged from 2.3 to $6.5 \%$ during a 3 -year study [14], and the annual incidence of new fractures was $0.8-2.6 \%$ in the 10-year Fracture REduction Evaluation of Denosumab in Osteoporosis every 6 Months (FREEDOM) study and extension [34]. However, the proportion of patients in the Czech Republic who experienced new fractures was higher $(6.0 \%)$ than that reported in clinical studies [14]. Differences in the baseline characteristics of the patients at the Czech Republic centers in our study and those of the FREEDOM study must be considered. The FREEDOM study included women aged 60-90 years who had a BMD $T$ score of -2.5 or less at the lumbar spine or total hip but no less than -4.0 at both sites; women who had taken oral bisphosphonates for more than 3 years were excluded [14]. In addition, differences in the reimbursement criteria in the Czech Republic and Slovakia mean that patients receiving denosumab in the Czech Republic were likely to have more severe disease than those in Slovakia. Indeed, in our study the proportion of patients who had had a previous osteoporotic fracture in the Czech Republic $(67.7 \%)$ was more than double that reported in Slovakia $(30.0 \%)$ or among participants in the FREEDOM trial (45.0\%) [35]. If previous fracture is considered a strong predictor of future osteoporotic fractures [36], a higher rate of new fractures would be expected in the Czech Republic population studied here. Given the severity of osteoporosis and the risk factors for fracture in the population in the Czech Republic in this study, the proportion of women experiencing new fractures could be considered relatively low.

No new safety signals were identified, and the ADRs that occurred during the study were consistent with the known safety profile of denosumab or reflected diseases that are common in older women [16, 17, 37, 38]. Given that more than half of patients from the Czech Republic were prescribed denosumab because of intolerance to previous osteoporosis medications, this suggests that denosumab has an acceptable safety profile in patients who are intolerant of other osteoporosis medications.

\section{Study Limitations}

Physicians actively chose to participate in this study and therefore may not be representative of all physicians in the Czech Republic and Slovakia. In addition, the patient population may not be representative of all women with osteoporosis in the countries studied. For example, willingness to participate in this study may indicate an increased interest in osteoporosis and an awareness of its seriousness; better adherence to therapy may therefore be expected in this group of patients compared with the general population. Only limited information was collected concerning individuals who chose not to participate, making it difficult to confirm that the patients studied 
were representative of the broader population treated with denosumab. Moreover, no information was collected on patients who refused denosumab treatment or the reasons for refusal. The study was also limited to centers in the Czech Republic and Slovakia, so the findings may not be representative of patient management practices in other European countries.

There were no mandatory study procedures, so the study was as close to routine clinical settings as possible. Repeat DXA assessments were likely to have been performed using a consistent scanner because most centers had access to only one machine, or chose to use the same machine for repeated scans of an individual patient, to enable a meaningful comparison of serial results. Nevertheless, because DXA assessments were not stipulated in the study protocol and there is no accepted goldstandard assessment interval in routine practice, our ability to assess improvements in BMD with denosumab treatment is limited. Furthermore, data on the least significant change for each DXA machine were not collected so it is not possible to comment on the precision of repeat measurements. As discussed above, the prospective observational nature of the study could have impacted the investigators' and patients' subjective response to treatment. Furthermore, this study was initiated soon after denosumab became available; the results may therefore reflect the experiences of the first patients receiving the drug in these countries (i.e., patients with severe disease who may be willing to receive a new therapy). Finally, the absence of a control group precludes formal comparisons of patient management and medication-taking behavior associated with denosumab and other osteoporosis treatments.

\section{CONCLUSION}

The results of this study show that the characteristics of patients prescribed denosumab differ between the Czech Republic and Slovakia, reflecting differences in reimbursement criteria; in both countries, the reasons for prescribing denosumab were in line with local reimbursement criteria. Denosumab was always administered by a healthcare professional, and more than $98 \%$ of patients received all their injections at the center where denosumab was initially prescribed. These patient management patterns may have contributed to the high proportion of individuals who received all four post-baseline injections during the 24-month follow-up. In both countries, most patients with a DXA scan performed on the same DXA machine at baseline and month 24 experienced an increase in their BMD $T$ score during the study period.

Furthermore, no new safety risks were identified, and ADRs were consistent with the known safety profile of denosumab. The findings of our study in patients who are at high risk for fracture are consistent with the growing body of evidence demonstrating the effectiveness of denosumab in real-world clinical practice.

\section{ACKNOWLEDGEMENTS}

The authors thank the participants for their participation in this study.

Funding. This study was sponsored by Amgen Inc. Article processing charges were funded by Amgen Inc. All authors had full access to all of the data in this study and take complete responsibility for the integrity of the data and accuracy of the data analysis.

Medical Writing, Editorial and Other Assistance. Editorial assistance in the preparation of this article was provided by Claire Desborough, MSc, of Amgen (Europe) $\mathrm{GmbH}$ (Cambridge, UK) and Kelly Soady, PhD, of Oxford PharmaGenesis (Oxford, UK). Funding for this support was provided by Amgen (Europe) GmbH.

Authorship. All named authors meet the International Committee of Medical Journal Editors (ICMJE) criteria for authorship for this article, take responsibility for the integrity of the work as a whole, and have given their approval for this version to be published. 
Disclosures. Soňa Tomková has received speaker fees from Amgen. Petr Kasalický has received speaker fees from Amgen. Zdenko Killinger has received speaker fees from Amgen. Lisa Hamilton was an employee of Amgen at the time of the study and holds stock. Lama Kalouche-Khalil was an employee of Amgen at the time of the study and holds stock. Lama Kalouche-Khalil is now an employee of SanofiGenzyme. Olga Růžičková and Roman Tyl have nothing to disclose.

Compliance with Ethics Guidelines. All procedures involving human participants were conducted in accordance with the ethical standards of the central regulatory ethics committee in Slovakia and with local institutional ethics committees in the Czech Republic (Nemocnice Havlickuv Brod-IRB/IEC Eticka komise Nemocnice Havlickuv Brod; Thomayerova nemocnice-IRB/IEC Eticka komise Institutu klinicke a experimentalni mediciny a Thomayerovy nemocnice; Revmatologicky ustavIRB/IEC Eticka Komise Revmatologickeho Ustavu; Nemocnice Ceske Budejovice as-IRB/ IEC Eticka Komise Nemocnice Ceske Budejovice). Study conduct was also in accordance with the 1964 Helsinki Declaration and its later amendments or comparable ethical standards. Informed consent was obtained from all individual participants included in the study.

Data Availability. There is a plan to share data. This may include de-identified individual patient data for variables necessary to address the specific research question in an approved data sharing request; also related data dictionaries, study protocol, statistical analysis plan, informed consent form, and/or clinical study report. Data sharing requests relating to data in this manuscript will be considered after the publication date and (1) this product and indication (or other new use) have been granted marketing authorization in both the US and Europe, or (2) clinical development discontinues and the data will not be submitted to regulatory authorities. There is no end date for eligibility to submit a data sharing request for these data. Qualified researchers may submit a request containing the research objectives, the
Amgen product(s) and Amgen study/studies in scope, endpoints/outcomes of interest, statistical analysis plan, data requirements, publication plan, and qualifications of the researcher(s). In general, Amgen does not grant external requests for individual patient data for the purpose of reevaluating safety and efficacy issues already addressed in the product labeling. A committee of internal advisors reviews requests. If not approved, a Data Sharing Independent Review Panel will arbitrate and make the final decision. Upon approval, information necessary to address the research question will be provided under the terms of a data sharing agreement. This may include anonymized individual patient data and/or available supporting documents containing fragments of analysis code where provided in analysis specifications. Further details are available at the following: http:// www.amgen.com/datasharing.

Open Access. This article is distributed under the terms of the Creative Commons Attribution-NonCommercial 4.0 International License (http://creativecommons.org/licenses/ by-nc/4.0/), which permits any noncommercial use, distribution, and reproduction in any medium, provided you give appropriate credit to the original author(s) and the source, provide a link to the Creative Commons license, and indicate if changes were made.

\section{REFERENCES}

1. Hernlund E, Svedbom A, Ivergard M, et al. Osteoporosis in the European Union: medical management, epidemiology and economic burden. A report prepared in collaboration with the International Osteoporosis Foundation (IOF) and the European Federation of Pharmaceutical Industry Associations (EFPIA). Arch Osteoporos. 2013;8:136.

2. Mediati RD, Vellucci R, Dodaro L. Pathogenesis and clinical aspects of pain in patients with osteoporosis. Clin Cases Miner Bone Metab. 2014;11:169-72.

3. Paolucci T, Saraceni VM, Piccinini G. Management of chronic pain in osteoporosis: challenges and solutions. J Pain Res. 2016;9:177-86.

4. Darba J, Kaskens L, Perez-Alvarez N, Palacios S, Neyro JL, Rejas J. Disability-adjusted-life-years 
losses in postmenopausal women with osteoporosis: a burden of illness study. BMC Public Health. 2015;15:324.

5. Tom SE, Adachi JD, Anderson FA Jr, et al. Frailty and fracture, disability, and falls: a multiple country study from the global longitudinal study of osteoporosis in women. J Am Geriatr Soc. 2013;61:327-34.

6. Kanis JA, Burlet N, Cooper C, et al. European guidance for the diagnosis and management of osteoporosis in postmenopausal women. Osteoporos Int. 2008;19:399-428.

7. Svedbom A, Hernlund E, Ivergard M, et al. Osteoporosis in the European Union: a compendium of country-specific reports. Arch Osteoporos. 2013;8:137.

8. Eriksen EF, Diez-Perez A, Boonen S. Update on longterm treatment with bisphosphonates for postmenopausal osteoporosis: a systematic review. Bone. 2014;58:126-35.

9. Vieira HP, Leite IA, Araujo Sampaio TM, et al. Bisphosphonates adherence for treatment of osteoporosis. Int Arch Med. 2013;6:24.

10. Cramer JA, Gold DT, Silverman SL, Lewiecki EM. A systematic review of persistence and compliance with bisphosphonates for osteoporosis. Osteoporos Int. 2007;18:1023-31.

11. Belhassen M, Confavreux CB, Cortet B, Lamezec L, Ginoux M, Van Ganse E. Anti-osteoporotic treatments in France: initiation, persistence and switches over 6 years of follow-up. Osteoporos Int. 2017;28:853-62.

12. Hadji P, Kyvernitakis I, Kann PH, et al. GRAND-4: the German retrospective analysis of long-term persistence in women with osteoporosis treated with bisphosphonates or denosumab. Osteoporos Int. 2016;27:2967-78.

13. Amgen. Prolia ${ }^{\circledR}$ Summary of Product Characteristics. 2010. http://www.ema.europa.eu/docs/en_GB/ document_library/EPAR_-_Product_Information/ human/001120/WC500093526.pdf. Accessed 31 Aug 2016.

14. Cummings SR, San Martin J, McClung MR, et al. Denosumab for prevention of fractures in postmenopausal women with osteoporosis. N Engl J Med. 2009;361:756-65.

15. Brown JP, Roux C, Ho PR, et al. Denosumab significantly increases bone mineral density and reduces bone turnover compared with monthly oral ibandronate and risedronate in postmenopausal women who remained at higher risk for fracture despite previous suboptimal treatment with an oral bisphosphonate. Osteoporos Int. 2014;25:1953-61.

16. Recknor C, Czerwinski E, Bone HG, et al. Denosumab compared with ibandronate in postmenopausal women previously treated with bisphosphonate therapy: a randomized open-label trial. Obstet Gynecol. 2013;121:1291-9.

17. Roux C, Hofbauer LC, Ho PR, et al. Denosumab compared with risedronate in postmenopausal women suboptimally adherent to alendronate therapy: efficacy and safety results from a randomized open-label study. Bone. 2014;58:48-54.

18. Hadji P, Papaioannou N, Gielen E, et al. Persistence, adherence, and medication-taking behavior in women with postmenopausal osteoporosis receiving denosumab in routine practice in Germany, Austria, Greece, and Belgium: 12-month results from a European non-interventional study. Osteoporos Int. 2015;26:2479-89.

19. Karlsson L, Lundkvist J, Psachoulia E, Intorcia M, Strom O. Persistence with denosumab and persistence with oral bisphosphonates for the treatment of postmenopausal osteoporosis: a retrospective, observational study, and a meta-analysis. Osteoporos Int. 2015;26:2401-11.

20. Lakatos P, Takacs I, Marton I, et al. A retrospective longitudinal database study of persistence and compliance with treatment of osteoporosis in Hungary. Calcif Tissue Int. 2016;98:215-25.

21. Silverman SL, Siris E, Kendler DL, et al. Persistence at 12 months with denosumab in postmenopausal women with osteoporosis: interim results from a prospective observational study. Osteoporos Int. 2015;26:361-72.

22. Migliaccio S, Francomano D, Romagnoli E, et al. Persistence with denosumab therapy in women affected by osteoporosis with fragility fractures: a multicenter observational real practice study in Italy. J Endocrinol Invest. 2017;40:1321-6.

23. Ross S, Samuels E, Gairy K, Iqbal S, Badamgarav E, Siris E. A meta-analysis of osteoporotic fracture risk with medication nonadherence. Value Health. 2011;14:571-81.

24. Slovak Republic Ministry of Health (2006) Guidelines for the diagnosis and treatment of osteoporosis. https://www.iofbonehealth.org/sites/default/files/ PDFs/National\%20Guidelines/Slovakia_OP_guide lines_2006.pdf Accessed 21 Feb 2017.

25. Rosa J, Šenk F, Palička V, et al. Diagnosis and treatment of postmenopausal osteoporosis. Statement of the Czech Society for Metabolic Bone Diseases. Osteol Bull. 2015;20:150-68. 
26. State Institute for Drug Control (2010) Prolia: pricing and reimbursement. http://www.sukl.eu/modules/ medication/detail.php? code $=0167653 \&$ tab $=$ prices $\&$ lang=2 Accessed 14 Aug 2017.

27. Vytrisalova M, Blazkova S, Palicka V, et al. Self-reported compliance with osteoporosis medicationqualitative aspects and correlates. Maturitas. 2008;60:223-9.

28. Tomkova S, Telepkova D, Vanuga P, et al. Therapeutic adherence to osteoporosis treatment. Int J Clin Pharmacol Ther. 2014;52:663-8.

29. Briot K, Ravaud P, Dargent-Molina P, Zylberman M, Liu-Leage S, Roux C. Persistence with teriparatide in postmenopausal osteoporosis; impact of a patient education and follow-up program: the French experience. Osteoporos Int. 2009;20:625-30.

30. Tamone C, Fonte G, Panico A, Molinatti PA, D'Amelio P, Isaia GC. Impact of a phone follow-up program on persistence with teriparatide or $\mathrm{PTH}(1-$ 84) treatment. Calcif Tissue Int. 2012;90:272-8.

31. Fahrleitner-Pammer A, Papaioannou N, Gielen E, et al. Factors associated with high 24-month persistence with denosumab: results of a real-world, non-interventional study of women with postmenopausal osteoporosis in Germany, Austria, Greece, and Belgium. Arch Osteoporos. 2017;12:58.

32. Fuksa L, Vytrisalova M. Adherence to denosumab in the treatment of osteoporosis and its utilization in the Czech Republic. Curr Med Res Opin. 2015;31:1645-53.

33. McCarney R, Warner J, Iliffe S, van Haselen R, Griffin M, Fisher P. The Hawthorne Effect: a randomised, controlled trial. BMC Med Res Methodol. 2007;7:30.

34. Bone HG, Wagman RB, Brandi ML, et al. 10 years of denosumab treatment in postmenopausal women with osteoporosis: results from the phase 3 randomised FREEDOM trial and open-label extension. Lancet Diabetes Endocrinol. 2017;5:513-23.

35. Palacios S, Kalouche-Khalil L, Rizzoli R, et al. Treatment with denosumab reduces secondary fracture risk in women with postmenopausal osteoporosis. Climacteric. 2015;18:805-12.

36. Kanis JA. Diagnosis of osteoporosis and assessment of fracture risk. Lancet. 2002;359:1929-36.

37. Brown JP, Prince RL, Deal C, et al. Comparison of the effect of denosumab and alendronate on BMD and biochemical markers of bone turnover in postmenopausal women with low bone mass: a randomized, blinded, phase 3 trial. J Bone Miner Res. 2009;24:153-61.

38. Miller PD, Pannacciulli N, Brown JP, et al. Denosumab or zoledronic acid in postmenopausal women with osteoporosis previously treated with oral bisphosphonates. J Clin Endocrinol Metab. 2016;101:3163-70. 